\title{
Impacto de las Cadenas Multinacionales de Comidas Rápidas en el Subsistema Papa
}

\author{
Mónica Mateos y Silvia Capezio ${ }^{1}$ \\ Resumen
}

Este documento examina el impacto inicial de la introducción de las cadenas de comidas rápidas multinacionales sobre la producción de papa en Argentina. Después de una breve descripción de las relaciones entre las cadenas de comidas rápidas y las que industrializan la papa, y de la industria de papa prefrita congelada en si, los autores estudian en más detalle el impacto de la industria sobre los productores. Entre otros resultados, se aprecia que en la producción para la industria los medianos productores participan más que los más grandes o los pequeños y que el proceso seguirá en el futuro diferenciando entre los tipos de productores. Además, las cadenas de comidas rápidas están impulsando un cambio en la estructura de la producción de papa en el país.

\section{Introducción}

La principal innovación de las cadenas de comidas rápidas multinacionales ha sido la industrialización de un servicio, cuyo principio de distinción es el carácter artesanal. En los años cincuenta, en los Estados Unidos, propusieron un modelo de consumo popular que en la presente década adquiere un carácter globalizado, al estar implantado en más de cien países, y particularmente en aquellos en desarrollo con modelos de consumo significativamente diferentes al de origen, como en los países asiáticos.

Estas cadenas se distinguen por brindar al consumidor los mismos productos con una calidad homogénea, siempre disponibles en una red de locales manejada mayormente en forma de franquicias, garantizando el mismo tipo de atención en establecimientos que se caracterizan por su limpieza, por un menú estándar, y un servicio rápido. En tal sentido, uno de los ejes de su éxito fue el desarrollo de una red de concesionarios y una de proveedores con una sofisticada infraestructura de abastecimiento, en vinculación con la industria procesadora a través de rígidas especificaciones, tratando siempre de reducir el costo de la materia prima. Los productos de origen agropecuario, constituyen uno de los principales insumos y por tanto estas cadenas han provocado un fuerte impacto en la industria alimentaria y en la producción primaria.

1 Facultad de Ciencias Agrarias, Universidad Nacional de Mar del Plata, Argentina.

Correo electrónico: mmateos@balcarce.inta.gov.ar y/o scapezio@balcarce.inta.gov.a 
El crecimiento del número de locales de este tipo de negocios en el MERCOSUR, fundamentalmente en Argentina y Brasil, fue el detonante de la entrada y crecimiento de la inversión directa extranjera en industrias dedicadas al abastecimiento de las cadenas de comida rápida a nivel regional. El objetivo del trabajo es comprender cómo se organiza este subsistema y su impacto en los distintos tipos de productores paperos en el sudeste bonaerense.

Este estudio forma parte de una investigación que se lleva a cabo desde 1995. En la metodología de trabajo se ha recurrido a información secundaria y entrevistas semiabiertas a: directivos y técnicos de industrias; informantes calificados; productores paperos e investigadores. En 1998/99 se entrevistaron en Argentina a directivos de una cadena multinacional de comidas rápidas y a una muestra de 18 productores de un listado de 101 productores de papa estratificados por volumen de papa contratado con la industria.

\section{Expansión de las cadenas multinacionales y el comercio de papa}

McDonalds es un ejemplo paradigmático de esta expansión, en 1975 las ventas fuera de los Estados Unidos alcanzaban el 8\%, en 1985 el $20 \%$ y en 1999 superaron el 50\%. Después de 20 años de su inicio se comienza a expandir a Canadá y el Caribe, Europa, Asia - Pacífico y en los ochenta a América del Sur. En 1999, las principales cadenas norteamericanas operaban aproximadamente 38,000 locales, de los cuales la mitad estaba fuera de Estados Unidos.

Alrededor del $90 \%$ de la papa prefrita congelada se destina a las cadenas de comidas rápidas (MSU, 1997), por tanto el comercio internacional de la primera se vincula fundamentalmente al proceso de internacionalización de las últimas. En el periodo 1990/98 se incrementa en más del doble el comercio de papas congeladas. Los exportadores netos son América del Norte y en mucha menor magnitud la Unión Europea (UE), vinculado al estancamiento de la demanda por la madurez del mercado de las cadenas de comidas. Los principales importadores son Japón, siguiéndole con menor importancia, pero con rápido crecimiento los países asiáticos en desarrollo (Filipinas, Indonesia, Taiwan, Malasia y Singapur), así como los de América Latina. Este último grupo cuenta en 1999 con el $6.6 \%$ de los locales McDonalds y el $4 \%$ de los de Burger King. Mientras el $63 \%$ de la primera operan en Brasil (7S mercado) y Argentina, la segunda opera el $54 \%$ en México y Puerto Rico, y el $10 \%$ en Argentina y Chile. Siguiendo este crecimiento aumentan las importaciones como se observa en el Figura 1. En el caso del MERCOSUR, en 1993 McDonalds cuenta 17 locales en Argentina y 100 en Brasil, en 1999 alcanzan a 205 y 920 unidades respectivamente 
Cuadro 1: Locales de las principales firmas multinacionales de comidas rápidas, 1986 y 1999

\begin{tabular}{|lllll|}
\hline & \multicolumn{3}{c}{1986} & \multicolumn{2}{c|}{1999} \\
\cline { 2 - 5 } Firma & Mundo & EEUU & Mundo & EEUU \\
\hline McDonalds & 9,000 & $78 \%$ & 26,800 & $47 \%$ \\
Burger King & 4,750 & $92 \%$ & 10,900 & $75 \%$ \\
\hline
\end{tabular}

Fuente: Elaboración propia, datos de páginas web de las firmas, y Love (1987).

En el Figura 1 se observa como Argentina pasa de importador de pequeña escala a exportador neto cuyo destino fundamental es Brasil, cubriendo un poco más del $50 \%$ de sus requerimientos, mostrando claramente la estrategia regional de la industria procesadora.

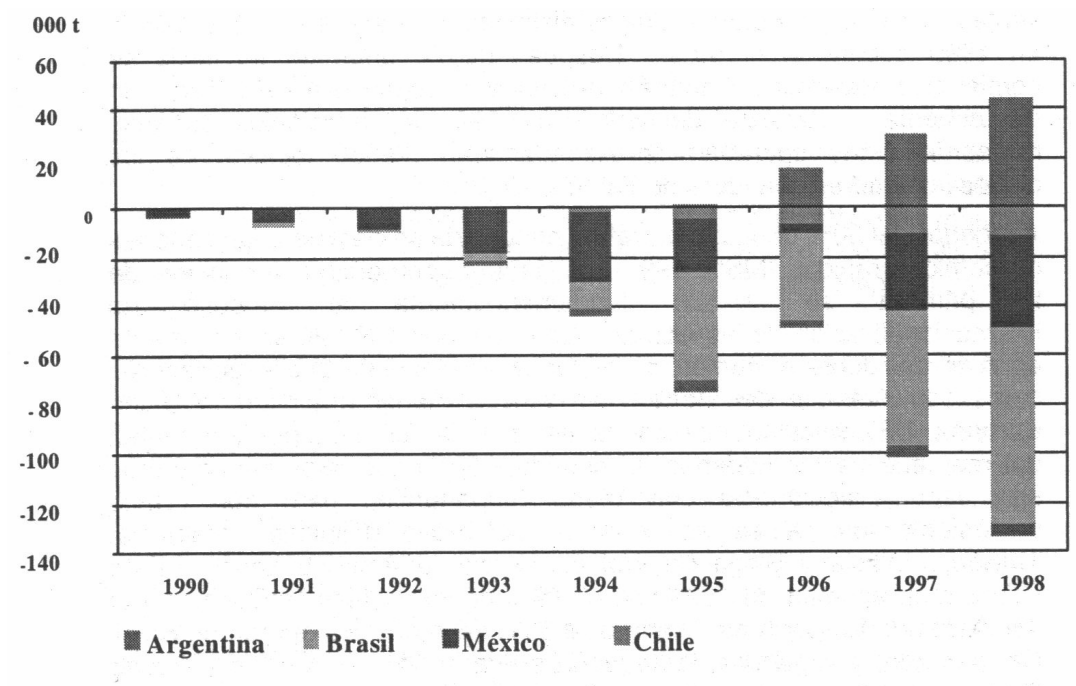

Fuente: Elaboración propia en base a datos de FAOSTAT.

Figura 1. Principales países importadores y exportadores de papa prefrita congelada, (0001) 


\section{Organización del abastecimiento de los restaurantes de comi- das rápidas en Argentina}

La empresa McDonalds con su expansión en 1995-96, produjo un gran cambio en su sistema de aprovisionamiento, en particular por la instalación de empresas proveedoras que siguen a su cliente en su proceso de expansión internacional, como parte de la globalización de los mercados, (Dunning, 1992). Se destaca la implantación de una empresa procesadora de papas y de otra que la abastece de hamburguesas y presta el servicio logístico, con un centro de distribución que recibe la mercadería de los proveedores y la entrega a los locales. Esto funciona de manera bastante semejante al sistema de producción justo en el momento, los locales hacen el pedido con una anticipación de 48hs para recibir el conjunto de la mercadería que ellos combinan y después comercializan.

Nagengast y Appleton (1997), señalan como los principales impactos de las cadenas de comidas rápidas en los subsistemas de papa, lácteos (mozzarella para pizzerías) y pollo (cadenas de pollo frito) en los Estados Unidos una serie de cambios significativos frente a la situación anterior (Cuadro 2).

Cuadro 2. Características de los subsistemas de abastecimiento

\begin{tabular}{|c|c|}
\hline Antes de las comidas rápidas & Con las comidas rápidas \\
\hline $\begin{array}{l}\text { Pequeñas granjas agrícolas } \\
\text { familiares }\end{array}$ & Grandes empresas agrícolas \\
\hline $\begin{array}{l}\text { Fijación del precio en los mercados } \\
\text { abiertos }\end{array}$ & $\begin{array}{l}\text { Precios establecidos en un } \\
\text { contrato }\end{array}$ \\
\hline Producción bajo una lógica de oferta & $\begin{array}{l}\text { Producción bajo una lógica } \\
\text { de demanda }\end{array}$ \\
\hline Múltiples mercados & $\begin{array}{l}\text { Menos mercados pero más } \\
\text { específicos }\end{array}$ \\
\hline Productos corrientes & Productos especiales \\
\hline Limitada dependencia tecnológica & Alta dependencia tecnológica \\
\hline $\begin{array}{l}\text { Escasos vínculos productor - } \\
\text { industria }\end{array}$ & $\begin{array}{l}\text { Importantes vínculos } \\
\text { productor-industria }\end{array}$ \\
\hline
\end{tabular}

Fuente: Elaborado para este estudio.

Esta síntesis de impactos muestra que la coordinación entre las distintas interfaces comidas rápidas/ industria, industria/ productor primario, no sólo ha modificado las formas de intercambio del mercado al contrato (forma híbrida) sino las condiciones de producción (Brousseau y Codron,1997; Jaffe,1992; Marión, 1985). Estos procesos se iniciaron en 
los Estados Unidos en los años '50 y se consolidaron en los 70 bajo determinadas condiciones históricas. Cómo se está desarrollando este proceso en Argentina es la cuestión central del presente trabajo.

\section{Interfase cadenas de comidas rápidas / industria de papa pre-frita congelada}

En el caso de papa, en un primer momento la empresa se abastecía de papa fresca y de importación de productos congelados, pasando luego al aprovisionamiento de la industria instalada localmente. Los proveedores, "socios" en el lenguaje de la empresa, deben firmar un compromiso de fórmula y, si bien realizan una proyección del volumen de compra anual, no firman contrato. Los principales requerimientos de las cadenas en papa prefrita son: cantidad en defectos; variedad; temperatura de entrega; cantidad de bastones por envase; rendimiento en el fritado. La negociación del precio se hace sobre "costos abiertos", es decir el proveedor debe detallar los componentes del costo a la cadena. Esta considera que están recibiendo producto de buena calidad, pero opinan que aún se pueden bajar los costos mejorando la eficiencia de la industria y disminuyendo el costo de la materia prima. La conformación de la interfase cliente/industria en un subsistema dominado por la lógica de mercado en fresco, introduce modificaciones sustanciales. En particular, es la cadena multinacional quien impone la lógica de acuerdos (formas híbridas) minimizando riesgos (en cantidad, calidad y precio) y coexiste con la «vía mercado» (se fija el precio por una cantidad mínima garantizada de compra y por encima de ese umbral se negocia por volumen).

\section{Interfase industria/ proveedores de la materia prima agrícola}

La industria de papa prefrita congelada en Argentina está conformada por dos empresas multinacionales especializadas y un pequeño número de empresas de carácter artesanal. La capacidad de procesamiento de las principales firmas es de alrededor de 72,000 $\mathrm{t}$ (una de 60,000 $\mathrm{t}$ y otra de $12,000 t)^{1}$. Teniendo en cuenta que el costo más importante para la industria es la materia prima, la firma dominante se ha localizado en el sudeste bonaerense, principal zona productora de papa.

En el Cuadro 3 se señalan los principales indicadores de la vinculación de la industria con los productores primarios. El crecimiento en el número de contratos fue muy significativo; de 35 contratos firmados en la campaña 94/95 se pasó a 85 en la siguiente y aproximadamente a 120 en 1997/98.

\footnotetext{
${ }^{1}$ La firma de mayor tamaño comenzó a fines de 1999 las obras para duplicar la capacidad y realizó también inversiones en instalaciones de recibo y almacenamiento de materia prima y producto procesado.
} 
Cuadro 3: Vinculación empresas /productores de papa (1997/98)

\begin{tabular}{|c|c|c|}
\hline Indicadores & Empresa X1 & Empresa X2 \\
\hline Realiza contratos & Desde 1994/95 & Desde 1996/97 \\
\hline $\begin{array}{l}\text { Abastecimiento con } \\
\text { contrato }\end{array}$ & $80-85 \%$ & $70-80 \%$ \\
\hline $\begin{array}{l}\text { Sup. estimada bajo } \\
\text { contrato }\end{array}$ & 4,500 ha & 900 ha \\
\hline Cantidad de contratos & 110 & $10-12$ \\
\hline Toneladas por contrato & Entre 250 y 7,0001 & Entre 250 y 5,0001 \\
\hline
\end{tabular}

Fuente: Elaboración propia en base a entrevistas.

Las principales condiciones de los contratos, principal innovación organizacional, se pueden observar en el Cuadro 4. Una forma de analizar y entender la multiplicidad de mecanismos utilizados en la coordinación entre agentes consiste en identificar en los acuerdos los mecanismos esenciales destinados a organizar la coordinación técnica, incentivar el esfuerzo, compartirlo y garantizar la realización de promesas (Brousseau, 1993). En tal sentido se propone una primera aproximación a un análisis dinámico (1994-1998) a través de la descomposición de los mismos en mecanismos de coordinación operativa (cantidad y fecha de entrega). La fecha de entrega vinculada al precio base se modificó de tres a ocho periodos, permitiendo una coordinación más ajustada a la demanda final.

Coordinación técnica y organizacional (condiciones de calidad y entrega de insumos básicos), en calidad se fueron agregando ítems que fueron resultado de la evaluación de cada campaña tanto en las condiciones mínimas como en las tolerancias. En insumos básicos se ha comenzado a exigir en la variedad tradicional el uso de semilla provista por la empresa, que para la mayoría de los productores constituye una desventaja por el mayor costo frente a su propia producción de semilla, mientras que para otros es una fuente de financiamiento. En relación a incentivos a la calidad (bonificaciones o descuentos) es donde la industria fue fijando cada vez más parámetros de acuerdo al seguimiento tecnológico que realiza a los mejores productores. Incentivos financieros (plazos de pago y financiamiento en equipamiento) se destaca el cumplimiento de los plazos de pago, a lo que se fue agregando financiamiento, lo que induce a vincularse con la industria y a cooperar con ella (Mateos,1998). De 1994 a la actualidad las condiciones de los contratos han ido cambiando paulatinamente, siempre hacia el aumento de las exigencias en calidad y la baja relativa en el precio que generalmente se 
ha situado por encima del precio de mercado.

Cuadro 4. Principales características de los contratos realizados por las industrias.

\begin{tabular}{|c|c|}
\hline Tema & Condiciones del contrato (1998/99) \\
\hline Cantidad & $\begin{array}{l}\text { En toneladas y en hectáreas para determinadas } \\
\text { variedades nuevas, identificando el lote a plantar. } \\
\text { La no entrega, implica el pago de lo contratado }\end{array}$ \\
\hline Recepción & $\begin{array}{l}8 \text { periodos de entrega, acordando luego la fecha de } \\
\text { entrada en planta. }\end{array}$ \\
\hline Precio base & $\begin{array}{l}\text { Variable, según fecha de entrega, varia entre US } \$ \\
126 \text { y US } \$ 166 \$ / t\end{array}$ \\
\hline $\begin{array}{l}\text { Condiciones } \\
\text { de calidad }\end{array}$ & $\begin{array}{l}\text { En \% menor a X en tamaño defectos y } \\
\text { enfermedades, condiciones mínimas y tolerancias, } \\
\text { se consideran siete ítems }\end{array}$ \\
\hline $\begin{array}{l}\text { Motivos de } \\
\text { rechazo }\end{array}$ & $\begin{array}{l}\text { En \% mayor a X en defectos de calidad, enfermeda- } \\
\text { des. Calidad de fritura, mínimo de MS, calidad } \\
\text { exigida por clientes. }\end{array}$ \\
\hline Bonificaciones & $\begin{array}{l}\text { Materia seca superior a } 20 \% \text {, se paga una suma fija } \\
\text { por tonelada, tiene } 5 \text { clases y tamaño mayor a } \\
90 \mathrm{~mm} \text { en la variedad Kennebec - Compensación en } \\
\text { el transporte por papa entregada y aceptada }\end{array}$ \\
\hline Descuentos & $\begin{array}{l}\text { Contenido de materia seca menor a } 17.9 \% \text { Tamaño } \\
\text { menor a } 90 \mathrm{~mm} \text { en el } 45 \% \text { de papa entregada } \\
\text { variedad Kennebec. Descuento por descarga en } \\
\text { papa entregada en bolsa. }\end{array}$ \\
\hline Plazo de pago & Dentro de los 30 días de la última entrega semanal \\
\hline $\begin{array}{l}\text { Entrega de } \\
\text { semilla }\end{array}$ & $\begin{array}{l}\text { Mínimo de } 50 \% \text { en todos los casos y algunos casos } \\
25 \% \text { de semilla Kennebec (sólo productores con } \\
\text { buena trayectoria de vinculación). }\end{array}$ \\
\hline Financiamiento & $\begin{array}{l}\text { Equipos de riego, equipos de plantación y } \\
\text { cosechadoras por leasing }\end{array}$ \\
\hline
\end{tabular}

Fuente: Elaboración propia en base a contratos suministrados por los productores.

\section{Tipos de productores y estrategias de vinculación con la industria}

El cultivo de papa se caracteriza por su relativa complejidad y alto costo en relación a los granos y a la ganadería (actividades predominantes en la región sudeste de Argentina). En tal sentido, los productores paperos se caracterizan por su conocimiento del cultivo; de la infraestructura, 
maquinarias y equipos específicos; arrendar la superficie a cultivar; integrar parte del proceso de comercialización y tener una actitud de mayor propensión al riesgo. Mosciaro (1996) concluyó que la forma predominante de organización social de la producción en papa es empresarial, teniendo en cuenta la dotación de capital y la utilización de mano de obra asalariada. Si bien era importante la heterogeneidad en niveles de capitalización, estrategias productivas y comerciales, el modelo principal está representado por el tipo papero grande que cultivaba más de 60 ha (62\% de los encuestados).

En el presente estudio, de los productores vinculados con la industria, el $80 \%$ son empresarios, y el resto son familiares empresariales (el cultivo de papa no es la actividad principal y sólo contratan mano de obra asalariada temporaria para las labores de riego, cortada, y cosecha). Por otra parte, la actividad es llevada por sociedades, en su mayoría de carácter familiar. Dada la complejidad del cultivo, esta organización simplifica el trabajo al dividir las tareas por chacras o por funciones.

El $90 \%$ de los productores realiza el cultivo bajo la forma de arrendamiento, sólo el $10 \%$ lo hace en superficie propia, ocupando algo menos del $10 \%$ de la superficie operada. Aproximadamente para el $50 \%$ de los productores, la papa es la actividad principal (cultivan entre el 60 y $100 \%$ de la superficie operada). Para la realización de las labores no recurren a los servicios de contratistas, a excepción de dos casos, que utilizaron este año un nuevo sistema de plantación-cosecha propuesto por una empresa, de todos modos lo hacen en un porcentaje mínimo de la superficie plantada. En este estudio, en la campaña 97/98, más del 50\% de los productores hizo contratos entre 250 t y 750 t y algo menos del $15 \%$ realizó contratos mayores a 2.000 t. Del volumen contratado por la industria, estos representaban el $30 \%$ y el $50 \%$ respectivamente.

En este estudio, la muestra fue seleccionada de una población estratificada por volúmenes contratados. Pero, teniendo en cuenta que para la mayoría de los productores el destino Industrial es complementario a la venta en fresco, para el análisis de la información estos fueron reagrupados por superficie operada con papa, ya que discriminando por toneladas contratadas no reflejaría las distintas estrategias de vinculación con la industria. En tal sentido, se identificaron cuatro tipos de productores según la superficie operada con papa: tipo $\mathrm{A}$, más de 700 ha (3 productores); tipo $B$, entre 400 y 700 ha (2 productores); tipo $\mathrm{C}$, entre 150 y 400 ha (6 productores) y tipo $\mathrm{D}$, menos de 150 ha (6 productores). Este análisis es una primera aproximación a una tipología de productores que entregan su producción a la industria teniendo en cuenta que el crecimiento del destino industrial de la misma es muy reciente.

El importante impacto de la industria se observa en la evolución de la superficie cultivada en el periodo (1995/99). Las hectáreas destinadas a la industria se duplicaron en dos años, mientras que la superficie total se 
incrementó en un $20 \%$. Sin embargo, la participación de los distintos tipos de productores en el volumen entregado a la industria muestra una importante variabilidad (Cuadro 5).

Cuadro 5. Participación (\%) de los tipos de productores en el volumen entregado a industria

\section{Tipo de productor}

\begin{tabular}{|llllll|}
\hline Campaña & A & B & C & D & Total en \\
\hline $95 / 96$ & 42 & 22 & 31 & 5 & 948 \\
$96 / 97$ & 35 & 22 & 37 & 6 & 1,638 \\
$97 / 98$ & 34 & 18 & 39 & 9 & 2,086 \\
$98 / 99$ & 39 & 0 & 52 & 9 & 1,831 \\
\hline
\end{tabular}

Fuente: Elaboración propia en base a entrevistas.

En el Cuadro 5 se observa que los productores del tipo $A$ han entregado a la industria entre 34 y $42 \%$ del volumen total; el tipo B con 18 y $22 \%$ de la papa entregada no realizó contratos en la última campaña; el tipo $C$ aumentó continuamente su participación de 31 a $52 \%$ y por último el tipo D, aumentó su participación, pero sin superar el 10\%.

\section{Tipo de productores y estrategias de vinculación con la industria}

El productor tipo A cultiva más de 700 ha de papa. Este grupo de grandes productores paperos (entre 800 y 1,700 ha) se destaca en primer lugar porque diversifica la actividad agropecuaria. Duplicó la superficie dedicada a industria mientras mantenía casi contante las hectáreas dedicadas a fresco y a semilla. En este grupo se observan dos estrategias claramente distintas de vinculación con la industria. Si bien en promedio la proporción aumentó de 14 a $23 \%$, uno de los productores, con una clara estrategia de expansión, duplicó en cuatro años la superficie total en papa, destinando un $50 \%$ de la misma a la industria. Esta estrategia en su opinión le permite cubrir los costos fijos de la superficie total operada con papa, permitiéndole solventar la expansión con menor riesgo.

En los otros dos casos, si bien ha aumentado la superficie operada con la industria, ésta no supera el $10 \%$ del total de papa. En tal sentido, operan con la industria para asegurarse efectivo en determinados meses del año, financiamiento bancario, y en equipos, y observan como desventaja la relación requerimientos de calidad / precios. Para estos productores la industria no representa una alternativa clara, en parte puede explicarse 
porque son reconocidos productores de papa en fresco, procesan la papa (lavada y "maquillada"), con lo cual obtienen un mejor precio en el mercado y en un caso se agrega una mayor diversificación de destinos aumentando en los últimos años las ventas a supermercados y la exportación.

El productor de papa tipo B cultiva entre 400 y 700 ha de papa. Este grupo está constituido por productores paperos arrendatarios. Comenzaron produciendo $33 \%$ de papa para la industria llegando al $40 \%$ en $97 / 98$. En la campaña $98 / 99$ no produjeron papa con este destino, disminuyendo las hectáreas totales plantadas en un $50 \%$ respecto al año anterior. Esto se debió a la falta de cumplimiento de los contratos y/o problemas financieros con la industria. En ambos casos vendían su producción al menos a dos empresas industriales y han tratado de diversificar los destinos de la producción en fresco (otras variedades para exportación o mercado interno). Este grupo es el que destina una mayor proporción para semilla. Los productores recibieron financiamiento de la industria en equipos e indirectamente financiamiento bancario (posesión de un contrato). Si bien son pocos casos, estos representan a un tipo de productores de la zona, que ya arrastraban una situación de descapitalización y fuerte endeudamiento lo que sumado a los bajos precios registrados en el mercado en los últimos años, ha contribuido a la disminución de la superficie 0 al abandonado de la producción. Este es uno de los problemas más señalados por la industria para lograr una red estable de abastecedores.

Los productores tipo C, cultivan entre 150 y 400 ha de papa y representan el grupo que más aumentó la superficie total de papa $(60 \%)$. Producen entre 40 y $100 \%$ con destino a la industria. Los que dedican el 100\% son quienes han expandido más la producción. En dos casos, el cultivo de papa representa menos de $20 \%$ de la superficie operada y en uno de ellos es el único que realiza el cultivo bajo propiedad. Evidentemente, con este tipo de productores la industria tiene actualmente mayores posibilidades de organizar su red de abastecimiento. Todos han recibido financiamiento en forma de equipos; señalan como mayor ventaja de la vinculación, el precio preestablecido y la seguridad de cobro del producto, lo cual les ha permitido incrementar la superficie en un cultivo de alto riesgo de mercado por los problemas de sobreproducción existentes. De todos modos, opinan que son muy altas las exigencias de calidad con relación al precio obtenido y en un caso se menciona que las variedades nuevas son más difíciles de producir.

Los paperos de tipo D, siembran menos de 150-ha de papa. Ellos se caracterizan por ser productores diversificados, su superficie operada con papa es menor de $20 \%$ y en la mayoría combinan superficie de su propiedad con aquella bajo arrendamiento. A diferencia del grupo anterior, los del grupo $\mathrm{D}$ dedican $50 \%$ o menos del cultivo de papa a la industria. En las últimas campañas mantuvieron las hectáreas totales dedicadas a papa, disminuyendo la proporción dedicada a fresco. La 
vinculación con la industria la consideran ventajosa porque les permite cubrir los costos totales, al garantizarle el precio y la seguridad de cobro. Sólo un tercio de los productores recibió financiamiento en equipos por parte de la industria e indirectamente préstamos bancarios. Este último aspecto puede visualizarse como un menor interés de la industria de vincularse con productores que cultivan menos de 50 ha con destino industrial. En todos los casos mencionan como la mayor desventaja de la industria las exigencias en calidad y la dificultad para producir las nuevas variedades.

\section{Impacto de la industria en innovaciones tecnológicas y organizacionales}

La industria introdujo innovaciones tecnológicas como nuevas variedades, tipo de riego, maquinaria de laboreo y plantación, cosechadoras para entrega a granel del producto. En cuanto a las variedades, la demanda de sus clientes exige cultivares de forma alargada y alto contenido de materia seca. La variedad Kennebec, cultivada tradicionalmente en la zona, si bien se adapta a las condiciones de fritado, no tiene la forma requerida, lo que hizo que se introdujeran dos nuevas variedades Russet Burbank.y Shepody. Una diferencia significativa para los productores es que mientras la variedad tradicional puede destinarse, aunque a menor precio, al mercado en fresco, la variedad Russet Burbank no es aceptada en el mercado por el tipo reticulado de piel. En tal sentido constituyen un activo específico que caracteriza la vinculación productor- industria (Williamson, 1994). Según la industria, las variedades introducidas aumentaron de $10 \%$ en 1994/95 hasta $60 \%$ en 1997/98. En esta investigación, en la campaña 1998/99 la proporción cultivada fue $60 \%$ con Kennebec y $40 \%$ con las variedades introducidas.

Con respecto al tipo de riego, la industria inicialmente propuso el pivote central, cuyo mayor inconveniente es la dificultad de traslado a lugares alejados, por lo cual no se adapta a un sistema de producción basado en el arrendamiento'. En la campaña 96/97, proponen equipos side roll. Estos presentan ventajas respecto al sistema convencional en la uniformidad de distribución del agua en la línea de riego, puede trasladarse y requiere poca mano de obra. Además cada equipo tiene una capacidad de 25 hectáreas y costo relativamente bajo. Las empresas los han entregado en forma de "leasing" (arrendamiento con opción a compra), a ser pagados en producto en un periodo de 6 años con una tasa de interés inferior a la de mercado. Su adopción ha sido generalizada en los grupos A, B y C, y en sólo el 30\% del grupo D. En cuanto al manejo del riego, si bien la industria aconseja utilizar distintos

${ }^{1}$ En el SE bonaerense los sistemas que se emplean para regar el cultivo de papa son de traslado manual y aspersores múltiples (convencional) y el cañón de aspersor único (de traslado manual o autopropulsado). Por estas características los productores pueden trasladarlos de chacra en chacra. 
métodos para detectar las necesidades de agua del cultivo, los productores manejan el riego en función de la capacidad del equipo que poseen.

La forma de cosecha predominante es la combinación manual / mecánica (76\%), y más del $90 \%$ de los productores entrega su producción en bolsas. Las cosechadoras en general son muy antiguas y golpean mucho la papa, pudiendo esto ser motivo de rechazo por la industria porque trae problemas en el almacenamiento. Por otra parte, los productores con la tendencia a la baja de los precios se han ido descapitalizando como para realizar grandes inversiones no suficientemente probadas, además de requerir trabajar una superficie importante para amortizar el equipamiento. En la campaña 1997/98 se entregaron en "leasing dos equipamientos completos para realizar cosecha y entrega a granel y maquinarias para preparación de suelo y plantación que se adaptan al sistema de cosecha. Estos fueron adquiridos por dos contratistas-productores, es decir fundamentalmente prestadores de servicios, disminuyendo el riesgo de amortización del equipo. El proceso de adopción es menor al previsto por las empresas, que tuvieron en cuenta las ventajas en cuanto a disminución de costos de la propuesta, pero no la situación socioeconómica de los productores. Estos dos equipos funcionaron a pleno en la campaña 1998/99, en la muestra sólo dos productores del grupo C los utilizaron en parte de la superficie operada.

En el manejo del cultivo los productores no registran diferencias importantes en cuanto al manejo de la producción con destino fresco o industria a excepción de la clasificación del cultivo poscosecha. Por otra parte, no se observan diferencias entre los distintos grupos, como tampoco en los resultados de la producción expresados en rendimiento. Pero sí se observaron algunas diferencias en el manejo de la producción.

El proceso de clasificación para la entrega del producto es diferencial para la industria ya que ésta es más exigente en cuanto a calidad del mismo. El descarte promedio para la industria oscila entre 15 y $25 \%$ mientras que para el destino en fresco es de $5 \%$ en papa sucia y $15 \%$ en papa lavada. En los últimos años los productores entrevistados han modificado la forma de clasificación tanto por las exigencias de la industria, como por los bajos precios de la papa en fresco. En este último caso, tratan de clasificar, cepillar y "maquillar" la papa, para obtener mejores precios.

Si bien no se registran diferencias notables entre el manejo del cultivo en fresco y aquel para la industria, a excepción del proceso de clasificación, las exigencias por parte de la industria van a conducir a un manejo más ajustado a este destino. 


\section{Conclusiones}

Entre los impactos más relevantes del rápido crecimiento de las cadenas multinacionales de comidas rápidas en el MERCOSUR, en el subsistema papa en Argentina se pueden mencionar:

\section{En la articulación cadenas/industria}

1. Las cadenas constituyen el eje de la demanda de papas prefritas congeladas, fijando a la industria requerimientos precisos de calidad, cantidad y tiempo de entrega.

2. Las empresas industriales multinacionales tradicionales, proveedoras en los mercados más importantes se instalaron en el MERCOSUR para abastecer el mercado regional. De esta manera, sustituyen importaciones y el abastecimiento de materia prima en fresco, disminuyendo los costos de transacción de las cadenas multinacionales ante la alternativa de importar producto.

3. La localización de la principal empresa en el sudeste bonaerense (importante zona productora del país) atiende a que la materia prima constituye el principal costo de la empresa.

\section{En la articulación industria/productores de papa}

1. Los requerimientos de calidad de la materia prima, involucran cambios en las formas de coordinación del subsistema, alterando no sólo las condiciones del intercambio sino también las de producción.

2. La coordinación vía contratos es expandida notablemente por la industria y actualmente involucra aproximadamente al 30\% de los productores y $20 \%$ de la superficie plantada en la región.

3. Los contratos incluyen mecanismos de coordinación operativa, coordinación técnica y organizacional, incentivos a la calidad e incentivos financieros. Estos han ido evolucionando hacia mayores requerimientos de calidad y menor precio, vinculados con las exigencias de las cadenas de comidas rápidas y con las evaluaciones continuas en las condiciones de producción del cultivo.

\section{En los productores de papa}

1. Los productores paperos tienen distintas estrategias de vinculación con la industria según la superficie total operada con papa (industria, fresco y semilla) y el grado de diversificación agrícola.

2. La introducción de innovaciones tecnológicas y organizacionales ha implicado un proceso continuo y rápido de aprendizaje de la industria y de los productores involucrados. 
3. Se ha demostrado en el trabajo las menores dificultades que enfrenta la industria en la introducción de nuevas variedades. Otras tecnologías recomendadas en una etapa inicial como riego, cosecha mecánica y entrega a granel debieron modificarse porque no se adaptaban a las condiciones de los productores, en particular por la utilización del arrendamiento y el alto grado de descapitalización de los empresarios.

4. El incipiente desarrollo de distintos prestadores de servicios especializados para destino industrial financiados por las empresas (en maquinaria para laboreo, plantación, cosecha y entrega a granel), prefiguran la aparición de nuevos o renovados actores en el subsistema tanto en los servicios como en la producción.

5. La industria está produciendo aceleradamente un proceso de diferenciación en los productores paperos.

Por último, el impacto de las cadenas de comidas rápidas en el subsistema papa no será tan claro, en el sentido que lo plantean Nagengast y Appleton (1997) para los ESTADOS UNIDOS, pero de todos modos reconfiguran la producción de papa en la región. La extensión del proceso puede ser menor, en parte porque el destino papa para este tipo de industria no va a tener la misma importancia que en los países desarrollados. En estos países el consumo fuera del hogar supera el $45 \%$ de los ingresos gastados en alimentos mientras en Argentina alcanza el $17 \%$. Por otra parte, el sentido de algunas transformaciones puede ser diferente, teniendo en cuenta las características específicas de la región en relación al predominio de las formas de organización social de la producción empresarial que producen bajo la forma de arrendamiento. Si a esto se suma el alto endeudamiento de los productores paperos y la expansión de prestadores de servicios que aplican las tecnologías recomendadas, la combinación de estas condiciones puede modificar profundamente los tipos actuales de productores que se articulan con la industria, favoreciendo la participación de grandes propietarios que se incorporen a la producción de papa bajo la lógica industrial.

\section{Bibliografía}

Brousseau, E. 1993. L'economies des contrats. PUF Paris.

Brousseau, E. y J.M. Codron, 1997. L'hybridation entre formes de gouvernance le cas de l'approvisionnement des grandes surfaces en fruits de contre saison. Colloque Grande Distribution Alimentaire, Sociéte Francaise d'Economie Rurale (SFER), 22-23. Montpellier. Mayo.

Dunning, J. 1992. Multinational enterprises and the global economy. Addison-Wesley England.

FAO STAT. 2000. Página web.Agricultura! data. Agricultural \& Food Trade. Crops \& livestock primary \& processed. 
Jaffe, S. 1992. How prívate entreprise organized agricultura! markets in Kenya. Policy Research Working Papers. WPS 823, World Bank , Washington, D.C.

Love, J. 1987. McDonald's. La empresa que cambió la forma de hacer negocios en el mundo. Grupo Editorial Norma. Colombia.

Marión, B. 1985. The organization and Performance of U.S. Food System. Lexington Books. Massachusetts.

Mateos, M. 1998. Análisis de las transformaciones de un subsistema alimentario en transición: el caso de la papa. Reunión Anual de la Asociación Argentina de Economía Agraria. La Plata. Octubre

Mosciaro, M. 1996. Caracterización de las formas de organización social en explotaciones paperas del Sudeste Bonaerense. Mimeo, Dpto. Economía y Sociología Rural, INTA, Balcarce.

Michigan State University (MSU). 1997. Demand and Supply assessment for the Michigan Frozen Potato Industry. Staff paper 97-47, Department of Agricultural Economics, Michigan State University, East Lansing Michigan.

Nagengast, Z. y C. Appleton,. 1997. "The quick service restaurant industry" en Schertz, L. y Daft, L. (eds.): Food and Agricultural:Markets: the quiet revolution. NPA Report N270, Washington.

Williamson, 0.1994. Les institutions de l'economie. Intereditions Paris. 